\title{
FROM HYPERSYMPLECTIC STRUCTURES TO COMPATIBLE PAIRS OF TENSORS ON A LIE ALGEROID
}

\author{
P. ANTUNES AND J.M. NUNES DA COSTA
}

\begin{abstract}
A hypersymplectic structure on a Lie algebroid determines several Poisson-Nijenhuis, $\Omega N$ and $P \Omega$ structures on that Lie algebroid. We show that these Poisson-Nijenhuis (respectively, $\Omega N, P \Omega$ ) structures on the Lie algebroid, are pairwise compatible.
\end{abstract}

\section{INTRODUCTION}

Pairs of tensor fields on manifolds, which are compatible in a certain sense, were studied by Magri and Morosi [7], in view of their application to integrable hamiltonian systems. These pairs determine Poisson-Nijenhuis ( $P N$ for short), $\Omega N$ and $P \Omega$ structures on the manifold where they are defined. The extension of these concepts to the Lie algebroid framework was done in [1] and [6]. Other related structures on Lie algebroids are the complementary forms [8] and the Hitchin pairs [4]. In [2], we studied the compatibility of pairs of these structures, where being compatible means that the sum of two structures of a certain type is still a structure of the same type.

Hypersymplectic structures on Lie algebroids were introduced in [1]. They are triples of symplectic forms on the Lie algebroid, satisfying some conditions. Taking the inverse of each symplectic form we get three Poisson bivectors and, if we consider the composition of the Poisson bivectors with the symplectic forms, we obtain $(1,1)$ tensors on the Lie algebroid, that turn to be Nijenhuis. In [1] it is proved that these symplectic forms, Poisson bivectors and Nijenhuis tensors, when considered in pairs, define $P N, \Omega N$ and $P \Omega$ structures on the Lie algebroid and that the symplectic forms $\omega_{i}$ are complementary forms. The aim of this Note is to show that the structures that we can derive from a given hypersymplectic structure on a Lie algebroid are, in most cases, pairwise compatible.

\section{Pairs of Tensors on Lie ALGEBroids}

Let $(A,[\cdot, \cdot], \rho)$ be a Lie algebroid over a manifold $M$. Frequently, we shall denote this Lie algebroid simply by $A$. Given a bivector $\pi$ and a 2 -form $\omega$ on $A$, we consider the usual bundle maps $\pi^{\#}: A^{*} \rightarrow A$ and $\omega^{b}: A \rightarrow A^{*}$ and the induced morphisms on sections, denoted by the same symbols, which are defined, for all $\alpha, \beta \in \Gamma\left(A^{*}\right)$ and $X, Y \in \Gamma(A)$, respectively by

$$
\left\langle\beta, \pi^{\#}(\alpha)\right\rangle=\pi(\alpha, \beta) \text { and }\left\langle Y, \omega^{b}(X)\right\rangle=\omega(X, Y)
$$

Key words and phrases. Lie algebroid; hypersymplectic; Poisson-Nijenhuis. 
A bivector $\pi$ on a Lie algebroid $(A,[\cdot, \cdot], \rho)$ is a Poisson bivector if $[\pi, \pi]=0$. Every bivector $\pi$ on a Lie algebroid $A$ defines a bracket on $\Gamma\left(A^{*}\right)$ :

$$
[\alpha, \beta]_{\pi}=\mathcal{L}_{\pi \#(\alpha)} \beta-\mathcal{L}_{\pi \#(\beta)} \alpha-\mathrm{d}(\pi(\alpha, \beta)),
$$

where $\mathrm{d}$ is the differential of the Lie algebroid $A$ and $\mathcal{L}$ is the Lie derivative determined by $\mathrm{d}$. In the case where $\pi$ is a Poisson bivector on $A$, the bracket $[\cdot, \cdot]_{\pi}$, given by $(1)$, is a Lie bracket and $\left(A^{*},[\cdot, \cdot]_{\pi}, \rho \circ \pi^{\#}\right)$ is a Lie algebroid.

Let $\pi$ be a Poisson bivector on $(A,[\cdot, \cdot], \rho)$. A 2 -form $\omega$ on $A$ is said to be a complementary form of $\pi$ if $[\omega, \omega]_{\pi}=0([8])$. In other words, $\omega$ is a Poisson bivector on the Lie algebroid $\left(A^{*},[\cdot, \cdot \cdot]_{\pi}, \rho \circ \pi^{\#}\right)$.

Let $N$ be a $(1,1)$-tensor on $(A,[\cdot, \cdot], \rho)$ and consider the deformed bracket $[\cdot, \cdot]_{N}$ on $\Gamma(A)$,

$$
[X, Y]_{N}=[N X, Y]+[X, N Y]-N[X, Y] .
$$

The Nijenhuis torsion of $N$ is the $(1,2)$-tensor $\mathcal{T} N$ given by

$$
\mathcal{T} N(X, Y)=[N X, N Y]-N[X, Y]_{N}, \quad X, Y \in \Gamma(A) .
$$

When the Nijenhuis torsion vanishes, $N$ is said to be a Nijenhuis tensor on $A$.

Let $\pi$ be a bivector on $A$ and $N$ a $(1,1)$-tensor, seen as a vector bundle map $N: A \rightarrow A$. If $N \circ \pi^{\#}=\pi^{\#} \circ N^{*}$, where $N^{*}$ denotes the transpose of $N, \pi_{N}$ given by $\pi_{N}(\alpha, \beta)=\pi\left(N^{*} \alpha, \beta\right), \alpha, \beta \in \Gamma\left(A^{*}\right)$, is a bivector on $A$. Analogously, if $\omega$ is a 2 -form on $A$ such that $\omega^{b} \circ N=N^{*} \circ \omega^{b}$, then $\omega_{N}$ given by $\omega_{N}(X, Y)=\omega(N X, Y)$, $X, Y \in \Gamma(A)$, is a 2 -form on $A$.

Let $\pi$ be a bivector and $N$ a $(1,1)$-tensor on a Lie algebroid $A$ such that $N \circ \pi^{\#}=$ $\pi^{\#} \circ N^{*}$. Recall that the Magri-Morosi concomitant $\mathcal{C}(\pi, N)$ of $\pi$ and $N$ is the $(2,1)$ tensor on $A$ defined by $[5,7]$ :

$$
\mathcal{C}(\pi, N)(\alpha, \beta)=\left([\alpha, \beta]_{\pi}\right)_{N^{*}}-[\alpha, \beta]_{\pi_{N}}, \quad \alpha, \beta \in \Gamma\left(A^{*}\right),
$$

where $[\cdot, \cdot]_{\pi_{N}}$ is the bracket $(1)$ determined by the bivector $\pi_{N}$ and $\left([\cdot, \cdot]_{\pi}\right)_{N^{*}}$ denotes the bracket $[\cdot, \cdot]_{\pi}$ deformed by $N^{*}$. From (1), (2) and (3), it is obvious that

$$
\mathcal{C}\left(\pi+\pi^{\prime}, N+N^{\prime}\right)=\mathcal{C}(\pi, N)+\mathcal{C}\left(\pi, N^{\prime}\right)+\mathcal{C}\left(\pi^{\prime}, N\right)+\mathcal{C}\left(\pi^{\prime}, N^{\prime}\right)
$$

for all bivectors $\pi, \pi^{\prime}$ and $(1,1)$-tensors $N, N^{\prime}$ on $A$.

Let $(A,[\cdot, \cdot], \rho)$ be a Lie algebroid, $\pi$ a bivector, $\omega$ a 2 -form and $N$ a $(1,1)$ tensor on $A$. The tensor fields $N, \pi$ and $\omega$, when taken in pairs, define well known structures on the Lie algebroid $A$. Next, we recall some of these structures.

A pair $(\pi, N)$ is a Poisson-Nijenhuis structure ( $P N$ structure, for short) on $(A,[\cdot, \cdot], \rho)$ if $\pi$ is Poisson, $N$ is Nijenhuis, $N \circ \pi^{\#}=\pi^{\#} \circ N^{*}$ and $\mathcal{C}(\pi, N)=0$.

A pair $(\omega, N)$ is an $\Omega N$ structure on $(A,[\cdot, \cdot], \rho)$ if $\omega$ is closed, $N$ is Nijenhuis, $\omega^{b} \circ N=N^{*} \circ \omega^{b}$ and $\omega_{N}$ is closed. If $\omega$ is symplectic, $\omega^{b} \circ N=N^{*} \circ \omega^{b}$ and $\omega_{N}$ is closed, the pair $(\omega, N)$ is a Hitchin pair [4].

A pair $(\pi, \omega)$ is a $P \Omega$ structure on $(A,[\cdot, \cdot], \rho)$ if $\pi$ is Poisson, $\omega$ is closed and $\omega_{N}$ is closed, where $N$ is the $(1,1)$-tensor on $A$ defined by $N=\pi^{\#} \circ \omega^{b}$.

A $P \Omega$ structure on a Lie algebroid determines a $P N$ and an $\Omega N$ structure on this Lie algebroid and is equivalent to a closed complementary form. More precisely, we have:

Proposition 1.1. [3, 6] Let $\pi$ and $\omega$ be, respectively, a Poisson bivector and a 2 -form on a Lie algebroid $(A,[\cdot, \cdot], \rho)$ and consider the $(1,1)$-tensor $N=\pi^{\#} \circ \omega^{b}$. 
(i) If $(\pi, \omega)$ is a P $\Omega$ structure on $A$, then $(\pi, N)$ and $(\omega, N)$ are, respectively, a $P N$ and an $\Omega N$ structure on $A$.

(ii) The pair $(\pi, \omega)$ is a $P \Omega$ structure on $A$ if and only if $\omega$ is a closed complementary form of $\pi$.

Let $N$ and $N^{\prime}$ be two $(1,1)$-tensors on a Lie algebroid $(A,[\cdot, \cdot], \rho)$ and $\left[N, N^{\prime}\right]_{F N}$ their Frölicher-Nijenhuis bracket which is defined, for all sections $X$ and $Y$ of $A$, by

$$
\begin{aligned}
{\left[N, N^{\prime}\right]_{F N}(X, Y)=} & {\left[N X, N^{\prime} Y\right]-N\left[X, N^{\prime} Y\right]-N^{\prime}[N X, Y]+N N^{\prime}[X, Y] } \\
& +\left[N^{\prime} X, N Y\right]-N^{\prime}[X, N Y]-N\left[N^{\prime} X, Y\right]+N^{\prime} N[X, Y] .
\end{aligned}
$$

Notice that $\left[N, N^{\prime}\right]_{F N}=\left[N^{\prime}, N\right]_{F N}$ and if $N=N^{\prime}$, then $\left[N, N^{\prime}\right]_{F N}=2 \mathcal{T} N$. So, if $N$ and $N^{\prime}$ are Nijenhuis tensors on $A$, we get

$$
\mathcal{T}\left(N+N^{\prime}\right)=0 \Leftrightarrow\left[N, N^{\prime}\right]_{F N}=0 .
$$

When the Nijenhuis tensors $N$ and $N^{\prime}$ satisfy (6), they are said to be compatible.

\section{HyPERSYMPLECTIC STRUCTURES ON LiE ALGEBROIDS}

In this section we recall, from [1] and [3], the notion of hypersymplectic structure on a Lie algebroid as well as their main properties, needed in the sequel.

Let $(A,[\cdot, \cdot], \rho)$ be a Lie algebroid and consider 3 symplectic forms $\omega_{1}, \omega_{2}$ and $\omega_{3}$ on $A$ with inverse Poisson bivectors $\pi_{1}, \pi_{2}$ and $\pi_{3}$, respectively. Then, for all $i \in\{1,2,3\}$, we have $\omega_{i}^{\mathrm{b}} \circ \pi_{i}^{\#}=\operatorname{Id}_{A^{*}}$ and $\pi_{i}^{\#} \circ \omega_{i}^{b}=\operatorname{Id}_{A}$. These $\omega_{i}$ and $\pi_{i}$ determine the transition (1,1)-tensors $N_{1}, N_{2}$ and $N_{3}$ on $A$, defined by

$$
N_{i}=\pi_{i-1}^{\#} \circ \omega_{i+1}^{b},
$$

where the indices of $\pi^{\#}$ and $\omega^{b}$ are considered as elements of $\mathbb{Z}_{3}$.

Definition 2.1. A triple $\left(\omega_{1}, \omega_{2}, \omega_{3}\right)$ of symplectic structures is an $\boldsymbol{\varepsilon}$-hypersymplectic structure on a Lie algebroid $(A,[\cdot, \cdot], \rho)$ if the transition $(1,1)$-tensors $N_{i}, i=1,2,3$, given by (7), satisfy $N_{i}^{2}=\varepsilon_{i} I d_{A}$, where the parameters $\varepsilon_{i}= \pm 1$ form the triple $\varepsilon=\left(\varepsilon_{1}, \varepsilon_{2}, \varepsilon_{3}\right)$.

Notice that $N_{i}^{-1}=\varepsilon_{i} N_{i}$, for all $i \in\{1,2,3\}$ and that

$$
\pi_{i-1}^{\#} \circ \omega_{i}^{b}=\varepsilon_{i+1} N_{i+1}
$$

with the indices in $\mathbb{Z}_{3}$.

Proposition 2.2. Let $\left(\omega_{1}, \omega_{2}, \omega_{3}\right)$ be an $\varepsilon$-hypersymplectic structure on a Lie algebroid $(A,[\cdot, \cdot], \rho)$. Then, $\left[N_{i}, N_{j}\right]_{F N}=0$ for all $i, j \in\{1,2,3\}$. In particular, $\mathcal{T} N_{i}=0, i=1,2,3$.

Recall that two Poisson bivectors $\pi$ and $\pi^{\prime}$ on a Lie algebroid $A$ are compatible if $\pi+\pi^{\prime}$ is a Poisson bivector on $A$ or, equivalently, if $\left[\pi, \pi^{\prime}\right]=0$. The Poisson bivectors $\pi_{i}, i=1,2,3$, of an $\varepsilon$-hypersymplectic structure are pairwise compatible, i.e.,

$$
\left[\pi_{i}, \pi_{j}\right]=0, \quad i, j \in\{1,2,3\} .
$$

Having an $\varepsilon$-hypersymplectic structure $\left(\omega_{1}, \omega_{2}, \omega_{3}\right)$ on a Lie algebroid $A$, we may define $g \in \bigotimes^{2} A^{*}$, through the vector bundle map $g^{b}$, by setting

$$
g^{b}=\varepsilon_{i-1} \varepsilon_{i+1} \omega_{i-1}^{b} \circ \pi_{i}^{\#} \circ \omega_{i+1}^{b},
$$


where the indices are taken in $\mathbb{Z}_{3}$. Moreover, $\left(g^{b}\right)^{*}=-\varepsilon_{1} \varepsilon_{2} \varepsilon_{3} g^{b}$, which means that, in the case where $\varepsilon_{1} \varepsilon_{2} \varepsilon_{3}=1, g$ is a 2 -form on $A$ and, consequently, $g^{-1}$ is a bivector on $A$. Notice that

$$
\left(g^{-1}\right)^{\#}=\varepsilon_{i-1} \varepsilon_{i+1} \pi_{i+1}^{\#} \circ \omega_{i}^{b} \circ \pi_{i-1}^{\#},
$$

with the indices taken in $\mathbb{Z}_{3}$.

Proposition 2.3. Let $\left(\omega_{1}, \omega_{2}, \omega_{3}\right)$ be an $\varepsilon$-hypersymplectic structure on a Lie algebroid $(A,[\cdot, \cdot], \rho)$, with $\varepsilon_{1} \varepsilon_{2} \varepsilon_{3}=1$. Then,

i) $g$ is a symplectic form on $A$;

ii) $g^{-1}$ is a Poisson bivector on $A$;

iii) $g^{-1}$ is compatible with $\pi_{i}$, i.e., $\left[\pi_{i}, g^{-1}\right]=0, i=1,2,3$.

From an $\varepsilon$-hypersymplectic structure on a Lie algebroid, we may derive several $P N, \Omega N$ and $P \Omega$ structures on that Lie algebroid, according to the next theorem.

Theorem 2.4. Let $\left(\omega_{1}, \omega_{2}, \omega_{3}\right)$ be an $\varepsilon$-hypersymplectic structure on a Lie algebroid $(A,[\cdot, \cdot], \rho)$. Then, for all $i, j \in\{1,2,3\}$,

i) the pairs $\left(\pi_{i}, N_{j}\right)$, with $i \neq j$, are $P N$ structures on $A$;

ii) the pairs $\left(\omega_{i}, N_{j}\right)$, with $i \neq j$, are $\Omega N$ structures on $A$;

iii) the pairs $\left(\pi_{i}, \omega_{j}\right)$, are $P \Omega$ structures on $A$.

Moreover, when $\varepsilon_{1} \varepsilon_{2} \varepsilon_{3}=1$,

iv) the pairs $\left(\pi_{i}, N_{i}\right)$ and $\left(g^{-1}, N_{i}\right)$, are $P N$ structures on $A$;

v) the pairs $\left(\omega_{i}, N_{i}\right)$ and $\left(g, N_{i}\right)$, are $\Omega N$ structures on $A$;

vi) the pairs $\left(\pi_{i}, g\right)$ and $\left(g^{-1}, \omega_{i}\right)$, are $P \Omega$ structures on $A$.

From Proposition 1.1 ii) we immediately deduce:

Corollary 2.5. If $\left(\omega_{1}, \omega_{2}, \omega_{3}\right)$ is an $\varepsilon$-hypersymplectic structure on $(A,[\cdot, \cdot], \rho)$, then $\omega_{i}$ is a complementary form of $\pi_{j}$, for all $i, j \in\{1,2,3\}$. In the case where $\varepsilon_{1} \varepsilon_{2} \varepsilon_{3}=1, g$ is a complementary form of $\pi_{i}$ and each $\omega_{i}$ is a complementary form of $g^{-1}, i=1,2,3$.

Remark 2.6. Notice that the 2 -forms of the $\Omega N$ structures in Theorem 2.4 ii) and $v)$ are symplectic. So, besides being $\Omega N$ structures, the pairs in the statements ii) and $v$ ) of that theorem, are also Hitchin pairs.

\section{Compatibility of Structures}

The compatibility of structures that are defined by pairs of tensors on a Lie algebroid was studied in [2].

Definition 3.1. Two $P N$ (respectively, $\Omega N, P \Omega$ ) structures on a Lie algebroid $A$ are said to be compatible if their sum is still a $P N$ (respectively, $\Omega N, P \Omega$ ) structure on A. Two complementary forms of a given Poisson bivector on $A$ are compatible if their sum is still a complementary form of the same Poisson bivector.

In what follows, we show that the $P N, \Omega N$ and $P \Omega$ structures determined by an $\varepsilon$-hypersymplectic structure on a Lie algebroid provide many examples of compatibility. In some cases, we also have the compatibility of complementary forms. 
3.1. The general case. Let $\left(\omega_{1}, \omega_{2}, \omega_{3}\right)$ be an $\varepsilon$-hypersymplectic structure on a Lie algebroid $(A,[\cdot, \cdot], \rho)$. Set, as before, $\pi_{i}^{\#}=\left(\omega_{i}^{b}\right)^{-1}$ and $N_{i}=\pi_{i-1}^{\#} \circ \omega_{i+1}^{b}$.

We start with the general case, i.e., the product $\varepsilon_{1} \varepsilon_{2} \varepsilon_{3}$ can be either 1 or -1 . In this case we know, from Theorem 2.4, that for $i, j \in\{1,2,3\}$, with $i \neq j$, the pairs $\left(\pi_{i}, N_{j}\right),\left(\omega_{i}, N_{j}\right)$ and $\left(\pi_{i}, \omega_{j}\right)$ are, respectively, $P N, \Omega N$ and $P \Omega$ structures on $A$. So, we have six structures of each type determined by the $\varepsilon$-hypersymplectic structure. Notice that the pairs $\left(\pi_{i}, \omega_{i}\right), i=1,2,3$, are also $P \Omega$ structures, but they are trivial in the sense that $\pi_{i}^{\#} \circ \omega_{i}^{b}=\operatorname{Id}_{A}$, so that they are not interesting from our point of view.

Proposition 3.2. With the indices taken in $\mathbb{Z}_{3}$, we have:

i) the PN structures $\left(\pi_{i}, N_{i-1}\right)$ and $\left(\pi_{i}, N_{i+1}\right)$ are compatible;

ii) the PN structures $\left(\pi_{i-1}, N_{i}\right)$ and $\left(\pi_{i+1}, N_{i}\right)$ are compatible.

Proof. i) From Proposition 2.2 and equation (6), we have that $N_{i-1}+N_{i+1}$ is a Nijenhuis tensor on $A$. Since the pairs $\left(\pi_{i}, N_{i-1}\right)$ and $\left(\pi_{i}, N_{i+1}\right)$ are $P N$ structures on $A$, it is obvious that the equality $\left(N_{i-1}+N_{i+1}\right) \circ \pi_{i}^{\#}=\pi_{i}^{\#} \circ\left(N_{i-1}+N_{i+1}\right)^{*}$ holds and also that $\mathcal{C}\left(\pi_{i}, N_{i-1}+N_{i+1}\right)=0$, see (4). Thus, i) is proved.

ii) We use (9) to get that $\pi_{i-1}+\pi_{i+1}$ is a Poisson bivector. Since the conditions $N_{i} \circ\left(\pi_{i-1}+\pi_{i+1}\right)^{\#}=\left(\pi_{i-1}+\pi_{i+1}\right)^{\#} \circ N_{i}^{*}$ and $\mathcal{C}\left(\pi_{i-1}+\pi_{i+1}, N_{i}\right)=0$ hold because $\left(\pi_{i-1}, N_{i}\right)$ and $\left(\pi_{i+1}, N_{i}\right)$ are $P N$ structures on $A$, the proof is complete.

Proposition 3.3. With the indices taken in $\mathbb{Z}_{3}$, we have:

i) the $\Omega N$ structures $\left(\omega_{i}, N_{i-1}\right)$ and $\left(\omega_{i}, N_{i+1}\right)$ are compatible;

ii) the $\Omega N$ structures $\left(\omega_{i-1}, N_{i}\right)$ and $\left(\omega_{i+1}, N_{i}\right)$ are compatible.

Proof. As we already observed in the proof of Proposition $3.2 \mathrm{i}), N_{i-1}+N_{i+1}$ is a Nijenhuis tensor on $A$. We have $\omega_{i}^{b} \circ\left(N_{i-1}+N_{i+1}\right)=\left(N_{i-1}+N_{i+1}\right)^{*} \circ \omega_{i}^{b}$, because $\omega_{i}^{b} \circ N_{i-1}=N_{i-1}^{*} \circ \omega_{i}^{b}$ and $\omega_{i}^{b} \circ N_{i+1}=N_{i+1}^{*} \circ \omega_{i}^{b}$. Since $\left(\omega_{i}\right)_{N_{i-1}+N_{i+1}}=$ $\left(\omega_{i}\right)_{N_{i-1}}+\left(\omega_{i}\right)_{N_{i+1}}$ and the 2-forms $\left(\omega_{i}\right)_{N_{i-1}}$ and $\left(\omega_{i}\right)_{N_{i+1}}$ are closed, the 2-form $\left(\omega_{i}\right)_{N_{i-1}+N_{i+1}}$ is also closed. This completes the proof of i). The proof of ii) is similar.

According to the observation in Remark 2.6, all the pairs in Proposition 3.3 are Hitchin pairs. Moreover, from i), we have that the Hitchin pairs $\left(\omega_{i}, N_{i-1}\right)$ and $\left(\omega_{i}, N_{i+1}\right)$ are compatible in the sense that $\left(\omega_{i}, N_{i-1}+N_{i+1}\right)$ is still a Hitchin pair.

Proposition 3.4. The $P \Omega$ structures $\left(\pi_{i-1}, \omega_{i}\right)$ and $\left(\pi_{i+1}, \omega_{i}\right)$ are compatible, where the indices are taken in $\mathbb{Z}_{3}$.

Proof. The sum $\pi_{i-1}+\pi_{i+1}$ is a Poisson bivector on $A$ (see (9)). From (7) and (8), we have $\left(\pi_{i+1}+\pi_{i-1}\right)^{\#} \circ\left(\omega_{i}\right)^{b}=N_{i-1}+\varepsilon_{i+1} N_{i+1}$. It remains to prove that $\left(\omega_{i}\right)_{N_{i-1}+\varepsilon_{i+1} N_{i+1}}$ is closed. Since, from Theorem 2.4 ii), the pairs $\left(\omega_{i}, N_{i-1}\right)$ and $\left(\omega_{i}, N_{i+1}\right)$ are $\Omega N$ structures, the 2 -forms $\left(\omega_{i}\right)_{N_{i-1}}$ and $\left(\omega_{i}\right)_{N_{i+1}}$ are closed. Thus, $\left(\omega_{i}\right)_{N_{i-1}+\varepsilon_{i+1} N_{i+1}}=\left(\omega_{i}\right)_{N_{i-1}}+\varepsilon_{i+1}\left(\omega_{i}\right)_{N_{i+1}}$ is closed.

3.2. The case $\varepsilon_{1} \varepsilon_{2} \varepsilon_{3}=1$. Now, we consider the case where we have an $\varepsilon$ hypersymplectic structure on a Lie algebroid $(A,[\cdot, \cdot], \rho)$, with $\varepsilon_{1} \varepsilon_{2} \varepsilon_{3}=1$. In this case, we know from Theorem $2.4 \mathrm{iv})$ that the pairs $\left(\pi_{i}, N_{i}\right)$ and $\left(g^{-1}, N_{i}\right), i=1,2,3$, are $P N$ structures on $(A,[\cdot, \cdot], \rho)$ and so the total number of $P N$ structures determined by the $\varepsilon$-hypersymplectic structure is twelve.

We may improve Proposition 3.2. 


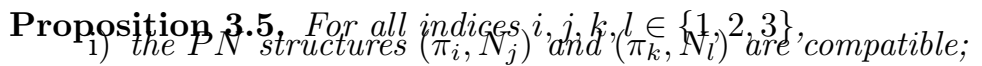

ii) the PN structures $\left(g^{-1}, N_{i}\right)$ and $\left(g^{-1}, N_{j}\right)$ are compatible;

iii) the PN structures $\left(g^{-1}, N_{i}\right)$ and $\left(\pi_{j}, N_{k}\right)$ are compatible.

Proof. i) As before, $\pi_{i}+\pi_{k}$ is a Poisson bivector and $N_{j}+N_{l}$ is a Nijenhuis tensor. Since $N_{i} \circ \pi_{j}^{\#}=\pi_{j}^{\#} \circ N_{i}^{*}$, the equality $\left(N_{j}+N_{l}\right) \circ\left(\pi_{i}+\pi_{k}\right)^{\#}=\left(\pi_{i}+\pi_{k}\right)^{\#} \circ\left(N_{j}+N_{l}\right)^{*}$ holds for all $i, j, k, l \in\{1,2,3\}$. Finally, from (4), we get $\mathcal{C}\left(\pi_{i}+\pi_{k}, N_{j}+N_{l}\right)=0$ and the proof is complete.

ii) The proof is similar to Proposition 3.2 i).

iii) From Proposition $2.3, g^{-1}+\pi_{j}$ is a Poisson bivector on $A$. The rest of the proof is similar to case i).

Summarizing, we have:

Theorem 3.6. Let $\left(\omega_{1}, \omega_{2}, \omega_{3}\right)$ be an $\varepsilon$-hypersymplectic structure on a Lie algebroid $(A,[\cdot, \cdot], \rho)$, with $\varepsilon_{1} \varepsilon_{2} \varepsilon_{3}=1$. Then, the twelve PN structures $\left(\pi_{i}, N_{j}\right),\left(g^{-1}, N_{k}\right)$, $i, j, k \in\{1,2,3\}$, on $A$, are pairwise compatible.

Concerning the $\Omega N$ structures obtained from an $\varepsilon$-hypersymplectic structure, with $\varepsilon_{1} \varepsilon_{2} \varepsilon_{3}=1$, we know, from Theorem $\left.2.4 \mathrm{v}\right)$, that the pairs $\left(\omega_{i}, N_{i}\right)$ and $\left(g, N_{i}\right)$, $i=1,2,3$, are $\Omega N$ structures. Thus, the total number of $\Omega N$ structures is twelve and we have:

Proposition 3.7. For all indices $i, j, k, l \in\{1,2,3\}$,

i) the $\Omega N$ structures $\left(\omega_{i}, N_{j}\right)$ and $\left(\omega_{k}, N_{l}\right)$ are compatible;

ii) the $\Omega N$ structures $\left(g, N_{i}\right)$ and $\left(g, N_{j}\right)$ are compatible;

iii) the $\Omega N$ structures $\left(g, N_{i}\right)$ and $\left(\omega_{j}, N_{k}\right)$ are compatible.

Thus, we get the following:

Theorem 3.8. Let $\left(\omega_{1}, \omega_{2}, \omega_{3}\right)$ be an $\varepsilon$-hypersymplectic structure on a Lie algebroid $(A,[\cdot, \cdot], \rho)$, with $\varepsilon_{1} \varepsilon_{2} \varepsilon_{3}=1$. Then, the twelve $\Omega N$ structures on $A,\left(\omega_{i}, N_{j}\right)$, $\left(g, N_{k}\right), i, j, k \in\{1,2,3\}$, are pairwise compatible.

Lastly we discussed the $P \Omega$ structures. It is known that, in the case of an $\varepsilon$ hypersymplectic structure with $\varepsilon_{1} \varepsilon_{2} \varepsilon_{3}=1$, the pairs $\left(\pi_{i}, g\right)$ and $\left(g^{-1}, \omega_{i}\right)$ are $P \Omega$ structures, for all $i \in\{1,2,3\}$ (see Theorem 2.4 vi)). Thus, the total number of nontrivial $P \Omega$ structures is twelve. Notice that the pair $\left(g^{-1}, g\right)$ is a trivial $P \Omega$ structure.

Proposition 3.9. For all indices $i, j, k, l \in\{1,2,3\}$,

i) the $P \Omega$ structures $\left(\pi_{i}, g\right)$ and $\left(\pi_{j}, g\right)$ are compatible;

ii) the $P \Omega$ structures $\left(\pi_{i}, g\right)$ and $\left(g^{-1}, \omega_{j}\right)$ are compatible;

iii) the $P \Omega$ structures $\left(\pi_{i}, g\right)$ and $\left(\pi_{j}, \omega_{k}\right)$ are compatible;

iv) the $P \Omega$ structures $\left(g^{-1}, \omega_{i}\right)$ and $\left(g^{-1}, \omega_{j}\right)$ are compatible;

v) the $P \Omega$ structures $\left(g^{-1}, \omega_{i}\right)$ and $\left(\pi_{j}, \omega_{k}\right)$ are compatible;

vi) the $P \Omega$ structures $\left(\pi_{i}, \omega_{j}\right)$ and $\left(\pi_{k}, \omega_{l}\right)$ are compatible;

vii) the trivial $P \Omega$ structure $\left(g^{-1}, g\right)$ is compatible with all the other $P \Omega$ structures.

Proof. i) From equation (9), $\pi_{i}+\pi_{j}$ is a Poisson bivector on $A$. We have $\pi_{i}^{\#} \circ g^{\mathrm{b}}=$ $\varepsilon_{i-1} \varepsilon_{i+1} N_{i+1} \circ \pi_{i}^{\#} \circ \omega_{i+1}^{b}=\varepsilon_{i-1} \pi_{i-1}^{\#} \circ \omega_{i+1}^{b}=\varepsilon_{i-1} N_{i}$. The 2 -forms $g_{\pi_{i}^{\#} \circ g^{b}}=\varepsilon_{i-1} g_{N_{i}}$ being closed, it is obvious that $g_{\left(\pi_{i}^{\#}+\pi_{j}^{\#}\right) \circ g^{b}}=\varepsilon_{i-1} g_{N_{i}}+\varepsilon_{j-1} g_{N_{j}}$ is also closed. 
ii) From Proposition 2.3, $\pi_{i}+g^{-1}$ is a Poisson bivector on $A$. From (11), (7) and (8) we get $\left(g^{-1}\right) \# \circ \omega_{j}^{b}=\varepsilon_{j-1} N_{j-1} \circ N_{j+1}$, which implies $N_{j} \circ\left(g^{-1}\right)^{\#} \circ \omega_{j}^{b}=\varepsilon_{j-1} \operatorname{Id}_{A}$, because $N_{j} \circ N_{j-1} \circ N_{j+1}=\operatorname{Id}_{A}$. But, since $N_{j}^{-1}=\varepsilon_{j} N_{j}$, we get $\left(g^{-1}\right)^{\#} \circ \omega_{j}^{b}=$ $\varepsilon_{j-1} \varepsilon_{j} N_{j}$. The pairs $\left(g, \varepsilon_{i-1} N_{i}\right)$ and $\left(\omega_{j}, \varepsilon_{j} N_{j}\right)$ being compatible $\Omega N$ structures (see Proposition 3.7), the 2-form $\left(g+\omega_{j}\right)_{\pi_{i}^{\#} \circ g^{b}+\left(g^{-1}\right) \# \circ \omega_{j}^{b}}=\left(g+\omega_{j}\right)_{\varepsilon_{i-1} N_{i}+\varepsilon_{j-1} \varepsilon_{j} N_{j}}$ is closed and the proof of ii) is complete.

iii) As in i), we have that $\pi_{i}+\pi_{j}$ is a Poisson bivector on $A$. For the rest of the proof, just notice that $\pi_{j}^{\#} \circ \omega_{k}$ is equal to $\operatorname{Id}_{A}$ if $j=k$, or is equal to $N_{j-1}$, if $k=j+1$, or $\varepsilon_{j+1} N_{j+1}$, if $k=j-1$. We also have $\pi_{i}^{\#} \circ g^{b}=\varepsilon_{i-1} N_{i}$. In any case, the 2 -form $\left(\omega_{k}+g\right)_{\pi_{i}^{\#} \circ g^{b}+\pi_{j}^{\#} \circ \omega_{k}^{b}}$ is closed because the pairs $\left(g, N_{i}\right)$ and $\left(\omega_{j}, N_{k}\right)$ are compatible $\Omega N$ structures.

The proofs of iv) - vi) are similar.

The Proposition 3.9 can be summarized as follows:

Theorem 3.10. Let $\left(\omega_{1}, \omega_{2}, \omega_{3}\right)$ be an $\varepsilon$-hypersymplectic structure on a Lie algebroid $(A,[\cdot, \cdot], \rho)$, with $\varepsilon_{1} \varepsilon_{2} \varepsilon_{3}=1$. Then, the sixteen $P \Omega$ structures $\left(\pi_{i}, \omega_{j}\right),\left(\pi_{k}, g\right)$, $\left(g^{-1}, \omega_{l}\right)$ and $\left(g^{-1}, g\right), i, j, k, l \in\{1,2,3\}$, on $A$, are pairwise compatible.

From Proposition 1.1, we get the following:

Corollary 3.11. Let $\left(\omega_{1}, \omega_{2}, \omega_{3}\right)$ be an $\varepsilon$-hypersymplectic structure on a Lie algebroid $(A,[\cdot, \cdot], \rho)$, with $\varepsilon_{1} \varepsilon_{2} \varepsilon_{3}=1$. Then, the complementary forms $g, \omega_{1}, \omega_{2}$ and $\omega_{3}$ of $\pi_{1}$, (respectively of $\pi_{2}$, of $\pi_{3}$ and of $g^{-1}$ ) are pairwise compatible.

\section{REFERENCES}

[1] P. Antunes, Crochets de Poisson gradués et applications: structures compatibles et généralisations des structures hyperkählériennes, Thèse de doctorat de l'École Polytechnique, March 2010.

[2] P. Antunes, J. M. Nunes da Costa, Nijenhuis and compatible tensors on Lie and Courant algebroids, arXiv:1207.1994.

[3] P. Antunes, J. M. Nunes da Costa, Hyperstructures on Lie algebroids, in preparation.

[4] M. Crainic, Generalized complex structures and Lie brackets, Bull. Braz. Math. Soc. (N.S.) 42 (2011), no. 4, 559-578.

[5] Y. Kosmann-Schwarzbach, F. Magri, Poisson-Nijenhuis structures, Ann. Inst. H. Poincaré, Phys. Théor. 53 (1990), no. 1, 35-81.

[6] Y. Kosmann-Schwarzbach, V. Rubtsov, Compatible structures on Lie algebroids and MongeAmpère operators, Acta Appl. Math., 109 (2010), no. 1, 101-135.

[7] F. Magri, C. Morosi, A geometrical characterization of integrable Hamiltonian systems through the theory of Poisson-Nijenhuis manifolds, Quaderno S 19, Univ. of Milan, 1984.

[8] I. Vaisman, Complementary 2-forms of Poisson structures, Compositio Mathematica 101 (1996) $55-75$.

Cmuc, Department of Mathematics, University of Coimbra, 3001-454 Coimbra, PorTUGAL, PANTUNES@MAT.UC.PT

CmuC, Department of Mathematics, University of Coimbra, 3001-454 Coimbra, PorTUGAL, JMCOSTA@MAT.UC.PT 